\title{
Finite-time stability of genetic regulatory networks with impulsive effects
}

\author{
Jianlong Qiuu, ${ }^{\mathrm{a}, *}$ Kaiyun Sun ${ }^{\mathrm{a}, \mathrm{d}}$, Chengdong Yang ${ }^{\mathrm{b}}$, Xiao Chen ${ }^{\mathrm{b}}$, Xiangyong Chen ${ }^{\mathrm{a}}$, Ancai Zhang ${ }^{\mathrm{c}}$ \\ ${ }^{a}$ School of Science, Linyi University, Linyi, Shandong 276005, China \\ ${ }^{b}$ School of Informatics, Linyi University, Linyi, Shandong 276005, China \\ ${ }^{c}$ School of Automobile, Linyi University, Linyi, Shandong 276005, China \\ ${ }^{d}$ School of Mathematical Sciences, Shandong Normal University, Jinan, Shandong 250014, China
}

\begin{abstract}
We study the finite-time stability of genetic regulatory networks with impulsive effects. Using the method of Lyapunov function, sufficient conditions of the finite-stability, in terms of linear matrix inequalities, are established. A numerical example is provided to further illustrate the significance of our results.
\end{abstract}

Keywords: impulsive genetic regulatory networks, finite-time stability, linear matrix inequalities approach, Lyapunov functions method

\section{Introduction}

It is well-known that the study of genetic regulatory networks (GRNs) has become a focus of interest in the past few years. It has been proved that many diseases are actually originated from the sicknesses of GRNs of the corresponding cell lineage, which mainly includes cancer, diabetes and AIDS. It is crucial to examine the dynamical behaviors of the GRNs. The stability of GRNs has been analyzed ([1, 2, 3, 4, 5, 6]) to some extent. GRNs illustrate the reciprocities among products of mRNA, genes and proteins, in terms of transcriptional and translational processes. It has been studied extensively over the last couple of decades through biological experiments (see $([7,8])$ for example). However, it is formidable to fully understand this kind of networks. One way is to set up mathematical models and perform rigorous mathematical analysis. Since time delays are ubiquitous in these processes and have influences on the stability of GRNs, it is indispensable in studying the effects from the time delays on the stability of GRNs. In 2002, L. Chen ([9]) presented a model for GRNs, which is described by delayed differential equations. Based on this model, some interesting results have been obtained $([10,11,12,13,14])$. Considering the environment fluctuations between intracellular and extracellular, we focus on the stability of impulsive GRNs with time-varying delays. It is widely accepted that the study of finite-time stability for networks is significant $([15,16,17])$. Meanwhile, the expression of gene and mRNA translated protein are accomplished in a much relatively short period of time. It is natural to consider the finite-time stability of GRNs. Some results have been obtained $([18,19,20])$. In our paper, we will study the finite-time stability of GRNs with impulsive effects and time-varying delays.

The paper is organized as follows: In section 2, we give a brief account of the model, problem formulation and some preliminaries. In section 3, we design a lyapunov function that consists of three parts, and derive sufficient conditions represented by linear matrix inequalities for the finite-time stability of GRNs with impulsive effects. In section 4 , we give a numerical example to demonstrate the significance of our result.

\section{Problem Formulation and some Preliminaries}

We study GRNs containing $n$ mRNAs and $n$ proteins represented by the differential equations as

$$
\left\{\begin{array}{l}
\dot{m}_{i}(t)=-k_{m i} m_{i}(t)+c_{i}\left(p\left(t-\tau_{p}(t)\right)\right) \\
\dot{p}_{i}(t)=-k_{p i} p_{i}(t)+r_{i} m_{i}\left(t-\tau_{m i}(t)\right)
\end{array}\right.
$$

${ }^{*}$ Corresponding author. Tel.:+86 0539 8766105; fax: +86 05398766105.

Email addresses: qjllinyi@aliyun.com (Jianlong Qiu ), sunkaiyun7646@126.com (Kaiyun Sun ), yangchengdong@lyu.edu.cn (Chengdong Yang), chx717@sina.com (Xiao Chen), xiangyongchen@lyu.edu.cn (Xiangyong Chen), zhangancai123@hotmail.com (Ancai Zhang)

(C) 2016. This manuscript version is made available under the Elsevier user license

http://www.elsevier.com/open-access/userlicense/1.0/ 
where $i=1,2, \ldots, n$, and $m_{i}(t) \in R_{+}^{n}$ denote the concentrations of mRNAs $i, p_{i}(t) \in R_{+}^{n}$ denote the concentrations of protein $i$. $k_{m i}$ and $k_{p i}$ are positive real numbers that describe the degradation rates for mRNAs $i$ and protein $i$, respectively. $r_{i}$ is a positive constant which indicate the rate of translating mRNAs $i$ to protein $i . c_{i}\left(p\left(t-\tau_{p}(t)\right)\right)$ is a non-linear function of $p_{1}\left(t-\tau_{p 1}(t)\right), \ldots, p_{n}\left(t-\tau_{p n}(t)\right)$ which present the regulation function of gene $i$. $\tau_{m i}(t)$ and $\tau_{p i}(t)$ are time delays for mRNAs $i$ and protein $i$, respectively.

In the translational process represented by the second equation in model $(1), r_{i} m_{i}(t)$ mean that one protein is produced by only one mRNA. In the transcriptional process described by the first equation in model (1), a gene or mRNA is usually influenced by multiple proteins. $c_{i}(p(t))$ is a function of the concentrations $p(t)$ of all possible proteins that represents the relative promoter or repressor activity of all possible proteins to gene $i$. In this paper, we take $c_{i}(p(t))=\sum_{j=1}^{n} c_{i j}\left(p_{j}(t)\right)$, where $c_{i j}\left(p_{j}(t)\right)$ is a function of the Hill form below,

$$
c_{i j}\left(p_{j}(t)\right)=a_{i j} \frac{1}{1+\left(p_{j}(t) / b_{j}\right)^{h_{j}}},
$$

assume that transcription factor $j$ is a repressor of gene $i$, or

$$
c_{i j}\left(p_{j}(t)\right)=a_{i j} \frac{\left(p_{j}(t) / b_{j}\right)^{h_{j}}}{1+\left(p_{j}(t) / b_{j}\right)^{h_{j}}},
$$

assume that transcription factor $j$ is an activator of gene $j$. Meanwhile $a_{i j}$ and $b_{j}$ are non-negative constants, and $h_{j}$ is the Hill coefficient that represent the degree of cooperativity. Suppose that $h_{j} \geq 1$ in this paper. So we have the following equation

$$
\frac{1}{1+\left(p_{j}(t) / b_{j}\right)^{h_{j}}}=1-\frac{\left(p_{j}(t) / b_{j}\right)^{h_{j}}}{1+\left(p_{j}(t) / b_{j}\right)^{h_{j}}}
$$

Then the system (1) change into the system as follows,

$$
\left\{\begin{array}{l}
\dot{m}_{i}(t)=-k_{m i} m_{i}(t)+\sum_{j=1}^{n} f_{i j} g_{j}\left(p_{j}\left(t-\tau_{p j}(t)\right)\right)+l_{i}, \\
\dot{p}_{i}(t)=-k_{p i} p_{i}(t)+r_{i} m_{i}\left(t-\tau_{m i}(t)\right),
\end{array}\right.
$$

where $i=1,2, \ldots, n . F=\left(f_{i j}\right)$ is an $n \times n$ matrix which represent regulatory relations of the network. Its definition can be described as:

$$
\begin{cases}f_{i j}=-a_{i j}, & j \text { is a repressor of gene } i, \\ f_{i j}=0, & j \text { does not regulate gene } i, \\ f_{i j}=a_{i j}, & j \text { is a activator of gene } i,\end{cases}
$$

Assume that Re is the set of all represses of gene $i$. So we define as $l_{i}=\sum_{j \in R e} a_{i j}$, where $l_{i}$ is a constant. Moreover, the function $g_{j}(\mu)$ can be written as the following form

$$
g_{j}(\mu)=\frac{\left(\mu / b_{j}\right)^{h_{j}}}{1+\left(\mu / b_{j}\right)^{h_{j}}}
$$

For variable $\mu$, it is clear that function $g_{j}(\mu)$ is monotonically increasing. When $h_{j} \geq 0$, it is obvious that the function $g_{j}(\mu)$ is continuously differential for $\mu \geq 0$. So, we get

$$
\eta_{j}=\max _{\mu \geq 0} g_{j}^{\prime}(\mu)=\frac{\left(h_{j}-1\right)^{\left(h_{j}-1\right) / h_{j}}\left(h_{j}+1\right)^{\left(h_{j}+1\right) / h_{j}}}{4 b_{j} h_{j}}>0
$$

Let us suppose that $(\bar{m}, \bar{p})$ is an equilibrium point of GRNs (2). Therefore, the following system are established

$$
\left\{\begin{array}{l}
0=-k_{m i} \bar{m}_{i}+\sum_{j=1}^{n} f_{i j} g_{j}\left(\bar{p}_{j}\right)+l_{i} \\
0=-k_{p i} \bar{p}_{i}+r_{i} \bar{m}_{i}
\end{array}\right.
$$

for $i=1,2, \ldots, n$. Let $x(t)=m(t)-\bar{m}$ and $y(t)=p(t)-\bar{p}$, we translate the equilibrium $(\bar{m}, \bar{p})$ into the origin. It is not difficult to get

$$
\left\{\begin{array}{l}
\dot{x}_{i}(t)=-k_{m i} x_{i}(t)+\sum_{j=1}^{n} f_{i j} \tilde{g}_{j}\left(y_{j}\left(t-\tau_{p j}(t)\right)\right)+l_{i}, \\
\dot{y}_{i}(t)=-k_{p i} y_{i}(t)+r_{i} x_{i}\left(t-\tau_{m i}(t)\right),
\end{array}\right.
$$


where $i=1,2, \ldots, n$, and $\tilde{g}_{j}\left(y_{j}\left(t-\tau_{p j}(t)\right)\right)=g_{j}\left(y_{j}\left(t-\tau_{p j}(t)\right)+\bar{p}_{j}\right)-g_{j}\left(\bar{p}_{j}\right)$. According to the inequation (4), we get

$$
0 \leq \tilde{g}_{j}\left(y_{j}\left(t-\tau_{p j}(t)\right)\right) \leq \eta_{j} y_{j}\left(t-\tau_{p j}(t)\right)
$$

In this study, we discuss the impulsive GRNs as follows:

$$
\begin{cases}\dot{x}(t)=-K_{m} x(t)+E \tilde{g}\left(y\left(t-\tau_{p}(t)\right)\right), & t \neq t_{k}, \\ \dot{y}(t)=-K_{p} x(t)+D x\left(t-\tau_{m}(t)\right) & , t \neq t_{k}, \\ \Delta x(t)=J_{k}\left(x\left(t_{k}^{-}\right)\right) & , t=t_{k}, \\ \Delta y(t)=H_{k}\left(y\left(t_{k}^{-}\right)\right) & , t=t_{k},\end{cases}
$$

where $\left.x(t)=\left[x_{1}(t), x_{2}(t), \ldots, x_{n}(t)\right]^{T}, y(t)=\left[y_{1}(t), y_{2}(t), \ldots, y_{n}(t)\right]^{T}, D=\operatorname{diag}\left(r_{1}, r_{2}, \ldots, r_{n}\right)\right), K_{m}=\operatorname{diag}\left(k_{m 1}, k_{m 2}, \ldots, k_{m n}\right)$, $K_{p}=\operatorname{diag}\left(k_{p 1}, k_{p 2}, \ldots, k_{p n}\right), E=\operatorname{diag}\left(\sum_{j=1}^{n} f_{i j}, \ldots, \sum_{j=1}^{n} f_{n j}\right), \tilde{g}(\cdot)=\left[\tilde{g}_{1}(\cdot), \tilde{g}_{2}(\cdot), \ldots, \tilde{g}_{3}(\cdot)\right]^{T}$, the time sequence $t_{k}$ satisfies $0<t_{0}<t_{1}<t_{2}<\cdots<t_{k}<t_{k+1}<\cdots$, and $\lim _{k \rightarrow \infty} t_{k}=\infty, J_{k}, H_{k} \in R^{n}$. These sequence $J_{k}, H_{k}$ are the effect of suddenly changing the state of system (6). Assume that $\Delta x=x\left(t_{k}^{+}\right)+x\left(t_{k}^{-}\right)$at fixed points sequence $t_{k}$, where $x\left(t_{k}^{+}\right)=\lim _{h \rightarrow 0^{+}} x\left(\tau_{k}+h\right)$ and $x\left(t_{k}^{-}\right)=\lim _{h \rightarrow 0^{+}} x\left(\tau_{k}-h\right)$. The quadratic from $\|x(t)\|_{Q}^{2}$ is definite as: $\|x(t)\|_{Q}^{2}=x^{T}(t) Q(t) x(t)$ for any state vector $x(t)$, and $Q(t)$ is non-negative definite matrix.

The initial value for the system (6) is : $x\left(t_{0}\right)=\varphi(t), y\left(t_{0}\right)=\psi(t)$.

Definition 1. Given a non-negative definite matrix $Q(t)$, two positive real numbers $c_{1}, c_{2}$, the system (6) is said to be finite-time stable with respect to $c_{1}, c_{2}, Q(t), T$ if and only if:

$$
\|\varphi(t)\|_{Q}^{2}+\|\psi(t)\|_{Q}^{2} \leq c_{1} \Longrightarrow\|x(t)\|_{Q}^{2}+\|y(t)\|_{Q}^{2} \leq c_{2}, \quad t \in[0, T]
$$

Assumption 1. $\tau_{p}(t)$ and $\tau_{m}(t)$ are time-varying delays satisfying

$$
\begin{aligned}
& 0 \leq \tau_{p 1} \leq \tau_{p}(t) \leq \tau_{p 2}, \quad \dot{\tau}_{p}(t) \leq \tau_{p 3}<\infty \\
& 0 \leq \tau_{m 1} \leq \tau_{m}(t) \leq \tau_{m 2}, \quad \dot{\tau}_{m}(t) \leq \tau_{m 3}<\infty
\end{aligned}
$$

Assumption 2. For $t \in\left[t_{0}, t_{0}+T\right]$, there exist positive-definite matrices $Q$, such that

$$
\begin{gathered}
\left\|J_{k}\left(x\left(t_{k}^{-}\right)\right)\right\|_{Q}^{2} \leq\left\|x\left(t_{k}^{-}\right)\right\|_{Q}^{2}, \\
\left\|H_{k}\left(x\left(t_{k}^{-}\right)\right)\right\|_{Q}^{2} \leq\left\|y\left(t_{k}^{-}\right)\right\|_{Q}^{2},
\end{gathered}
$$

Lemma 1. If $M \in R^{(n \times n)}$ is a positive definite matrix, $N \in R^{(n \times n)}$ is a symmetric matrix, then

$$
\lambda_{\min }\left(M^{-1} N\right) x^{T} M x \leq x^{T} N x \leq \lambda_{\max }\left(M^{-1} N\right) x^{T} M x
$$

Lemma 2. For any positive definite matrix $P \in R^{n \times n}$, there exist a scalar $q>0$ and a vector-valued function $\rho:[0, q] \rightarrow R^{n}$ such that

$$
\left(\int_{0}^{q} \rho(s) d s\right)^{T} P\left(\int_{0}^{q} \rho(s) d s\right) \leq q \int_{0}^{q} \rho^{T}(s) P \rho(s) d s .
$$

\section{Main Results}

Theorm 1. For impulsive networks (6) with Assumptions 1 and 2, given a scalar $\alpha>0$, if there exist symmetric positive definite matrices $P_{1}, P_{2}, G_{1}, R_{1}, R_{2}, R_{3}, R_{4}$, the diagonal matrix $\Gamma_{m}=\operatorname{diag}\left(\gamma_{m 1}, \ldots, \gamma_{m n}\right) \geq 0, m=1,2$, matrices $G_{2}, G_{3}, M_{1}$, and scalars $\lambda_{1}, \lambda_{2}$ such that the following inequalities

$$
\begin{aligned}
& {\left[\begin{array}{cc}
G_{2} & M_{1} \\
* & G_{3}
\end{array}\right] \geq 0} \\
& \Phi=\left(\begin{array}{cccccccccc}
a_{11} & 0 & 0 & 0 & 0 & 0 & 0 & 0 & 0 & a_{1,10} \\
& -R_{1}-R_{2} & 0 & 0 & 0 & 0 & 0 & 0 & 0 & 0 \\
* & a_{33} & 0 & 0 & 0 & 0 & 0 & 0 & 0 \\
* & * & -R_{2} & 0 & 0 & 0 & 0 & 0 & 0 \\
& * & * & * & a_{55} & 0 & 0 & 0 & M_{1}+\Gamma_{1} N & 0 \\
* & * & * & * & -R_{3}-R_{4} & 0 & 0 & 0 & 0 \\
* & * & * & * & * & a_{77} & 0 & 0 & a_{7,10} \\
& * & * & * & * & * & * & -R_{4} & 0 & 0 \\
& * & * & * & * & * & * & * & G_{3}-2 \Gamma_{1} & 0 \\
& * & * & * & * & * & * & * & * & a_{10,10}
\end{array}\right)<0
\end{aligned}
$$


where $a_{11}=-2 P_{1} K_{m}+G_{1}+\tau_{m 1}^{2} K_{m}^{T} R_{1} K_{m}+\left(\tau_{m 2}-\tau_{m 1}\right)^{2} K_{m}^{T} R_{2} K_{m}-R_{1}-\alpha P_{1}, a_{33}=-G_{1}\left(1-\tau_{m 3}\right)+\tau_{p 1}^{2} D^{T} R_{3} D+$ $\left(\tau_{p 2}-\tau_{p 1}\right)^{2} D^{T} R_{4} D-2 R_{2}, a_{55}=-2 P_{2} K_{p}+G_{2}+\tau_{p 1}^{2} K_{p}^{T} R_{3} K_{p}+\left(\tau_{p 2}-\tau_{p 1}\right)^{2} K_{p}^{T} R_{4} K_{p}-R_{3}-\alpha P_{2}, a_{77}=-G_{2}(1-$ $\left.\tau_{p 3}\right)-2 R_{4}, a_{10,10}=-G_{3}\left(1-\tau_{p 3}\right)+\tau_{m 1}^{2} E^{T} R_{1} E+\left(\tau_{m 2}-\tau_{m 1}\right)^{2} E^{T} R_{2} E-2 \Gamma_{2}, a_{1,10}=P_{1} E-\tau_{m 1}^{2} E^{T} R_{1} K_{m}-\left(\tau_{m 2}-\right.$ $\left.\tau_{m 1}\right)^{2} E^{T} R_{2} K_{m}, a_{7,10}=M_{1}\left(1-\tau_{p 3}\right)+\Gamma_{2} U,\left(\lambda_{1} / \lambda_{2}\right) e^{\alpha T} c_{1} \leq c_{2}$, and $\lambda_{1}=\max \left\{\lambda_{\max }\left(Q^{-1} P_{1}\right)+\tau_{m 2} \lambda_{\max }\left(Q^{-1} G_{1}\right)+\right.$ $1 / 2\left[\tau_{m 1}^{3} \lambda_{\max }\left(Q^{-1} R_{1}\right)+\left(\tau_{m 2}-\tau_{m 1}\right)^{3} \lambda_{\max }\left(Q^{-1} R_{2}\right)\right], \tau_{p 2}\left(\lambda_{\max }\left(Q^{-1} G_{2}\right)+\eta^{2} \lambda_{\max }\left(Q^{-1} G_{3}\right)+2 \eta \lambda_{\max }\left(Q^{-1} M_{1}\right)\right)+\lambda_{\max }\left(Q^{-1} P_{2}\right)+$ $\left.1 / 2\left[\tau_{p 1}^{3} \lambda_{\max }\left(Q^{-1} R_{3}\right)+\left(\tau_{p 2}-\tau p 1\right)^{3} \lambda_{\max }\left(Q^{-1} R_{4}\right)\right]\right\}, \eta=\max \eta_{i}, i=1,2, \ldots, n . \lambda_{2}=\min \left[\left(\lambda_{\min }\left(Q^{-1} P_{1}\right), \lambda_{\min }\left(Q^{-1} P_{2}\right)\right]\right.$, then, the system (6) is finite time stable with respect to positive real numbers $\left(c_{1}, c_{2}, T\right)$.

proof. According to the system (6), we construct the Lyapunov-krasovskii functional as follows:

$$
V(t)=V_{1}(t)+V_{2}(t)+V_{3}(t)
$$

where

$$
\begin{aligned}
V_{1}(t)= & x^{T}(t) P_{1} x(t)+y^{T}(t) P_{2} y(t), \\
V_{2}(t)= & \int_{t-\tau_{m}(t)}^{t} x^{T}(s) G_{1} x(s) d s+\int_{t-\tau_{p}(t)}^{t}\left[y^{T}(s), \tilde{g}^{T}(y(s))\right]\left[\begin{array}{cc}
G_{2} & M_{1} \\
* & G_{3}
\end{array}\right] \times\left[\begin{array}{c}
y(s) \\
\tilde{g}(y(s))
\end{array}\right] d s, \\
V_{3}(t)= & \int_{-\tau_{m 1}}^{0} \int_{t+\theta}^{t} \tau_{m 1} \dot{x}^{T}(s) R_{1} \dot{x}(s) d s d \theta+\int_{-\tau_{m 2}}^{-\tau_{m 1}} \int_{t+\theta}^{t}\left(\tau_{m 2}-\tau_{m 1}\right) \dot{x}^{T}(s) R_{2} \dot{x}(s) d s d \theta \\
& +\int_{-\tau_{p 1}}^{0} \int_{t+\theta}^{t} \tau_{p 1} \dot{y}^{T}(s) R_{3} \dot{y}(s) d s d \theta+\int_{-\tau_{p 2}}^{-\tau_{p 1}} \int_{t+\theta}^{t}\left(\tau_{p 2}-\tau_{p 1}\right) \dot{y}^{T}(s) R_{4} \dot{y}(s) d s d \theta,
\end{aligned}
$$

take the derivatives of $V_{i}$ on the interval $t \in\left(t_{0}, t_{1}\right)$, we have

$$
\begin{aligned}
\dot{V}_{1}(t)= & 2 x^{T}(t) P_{1} \dot{x}(t)+2 y^{T}(t) P_{2} \dot{y}(t), \\
\dot{V}_{2}(t)= & x^{T}(t) G_{1} x(t)-x^{T}\left(t-\tau_{p}(t)\right) G_{1} x^{T}\left(t-\tau_{m}(t)\right)\left(1-\dot{\tau}_{m}(t)\right)+\left[y^{T}(s), \tilde{g}^{T}(y(s))\right]\left[\begin{array}{cc}
G_{2} & M_{1} \\
* & G_{3}
\end{array}\right]\left[\begin{array}{c}
y(t) \\
\tilde{g}(y(t))
\end{array}\right] \\
& -\left[y^{T}\left(t-\tau_{p}(t)\right), \tilde{g}^{T}\left(y\left(t-\tau_{p}(t)\right)\right)\right]\left[\begin{array}{cc}
G_{2} & M_{1} \\
* & G_{3}
\end{array}\right]\left[\begin{array}{c}
y\left(t-\tau_{p}(t)\right) \\
\tilde{g}\left(y\left(t-\tau_{p}(t)\right)\right)
\end{array}\right]\left(1-\dot{\tau}_{p}(t)\right), \\
V_{3}(t)= & \tau_{m 1}^{2} \dot{x}^{T}(t) R_{1} \dot{x}(t)-\int_{t-\tau_{m 1}}^{t} \tau_{m 1} \dot{x}^{T}(\theta) R_{1} \dot{x}(\theta) d \theta+\left(\tau_{m 2}-\tau_{m 1}\right)^{2} \dot{x}^{T}(t) R_{2} \dot{x}(t) \\
& -\int_{t-\tau_{m 2}}^{t-\tau_{m 1}}\left(\tau_{m 2}-\tau_{m 1}\right) \dot{x}^{T}(\theta) R_{2} \dot{x}(\theta) d \theta+\tau_{p 1}^{2} \dot{y}^{T}(t) R_{3} \dot{y}(t)-\int_{t-\tau_{p 1}}^{t} \tau_{p 1} \dot{y}^{T}(\theta) R_{3} \dot{y}(\theta) d \theta \\
& +\left(\tau_{p 2}-\tau_{p 1}\right)^{\dot{y}^{T}} \dot{y}^{T}(t) R_{4} \dot{y}(t)-\int_{t-\tau_{p 2}}^{t-\tau_{p 1}}\left(\tau_{p 2}-\tau_{p 1}\right) \dot{y}^{T}(\theta) R_{4} \dot{y}(\theta) d \theta .
\end{aligned}
$$

By lemma2, we get

$$
\begin{aligned}
-\int_{t-\tau_{m 1}}^{t} \tau_{m 1} \dot{x}^{T}(\theta) R_{1} \dot{x}(\theta) d \theta \leq & -\left[x(t)-x\left(t-\tau_{m 1}\right)\right]^{T} R_{1}\left[x(t)-x\left(t-\tau_{m 1}\right)\right] \\
-\int_{t-\tau_{p 1}}^{t} \tau_{p 1} \dot{y}^{T}(\theta) R_{3} \dot{y}(\theta) d \theta \leq & -\left[y(t)-y\left(t-\tau_{p 1}\right)\right]^{T} R_{1}\left[y(t)-y\left(t-\tau_{p 1}\right)\right] \\
-\int_{t-\tau_{m 2}}^{t-\tau_{m 1}}\left(\tau_{m 2}-\tau_{m 1}\right) \dot{x}^{T}(\theta) R_{2} \dot{x}(\theta) d \theta= & -\left(\tau_{m 2}-\tau_{m 1}\right)\left[\int_{t-\tau_{m 2}}^{t-\tau_{m}(t)} \dot{x}^{T}(\theta) R_{2} \dot{x}(\theta) d \theta+\int_{t-\tau_{m}(t)}^{t-\tau_{m 1}} \dot{x}^{T}(\theta) R_{2} \dot{x}(\theta) d \theta\right] \\
= & -\int_{t-\tau_{m 2}}^{t-\tau_{m}(t)}\left(\tau_{m 2}-\tau_{m}(t)\right) \dot{x}^{T}(\theta) R_{2} \dot{x}(\theta) d \theta-\int_{t-\tau_{m}(t)}^{t-\tau_{m 1}}\left(\tau_{m}(t)-\tau_{m 1}\right) \dot{x}^{T}(\theta) R_{2} \dot{x}(\theta) d \theta \\
& -\int_{t-\tau_{m 2}}^{t-\tau_{m}(t)}\left(\tau_{m}(t)-\tau_{m 1}\right) \dot{x}^{T}(\theta) R_{2} \dot{x}(\theta) d \theta-\int_{t-\tau_{m}(t)}^{t-\tau_{m 1}}\left(\tau_{m 2}-\tau_{m}(t)\right) \dot{x}^{T}(\theta) R_{2} \dot{x}(\theta) d \theta
\end{aligned}
$$




$$
\begin{aligned}
\leq & -\left[x\left(t-\tau_{m}(t)\right)-x\left(t-\tau_{m 2}\right)\right]^{T} R_{2}\left[x\left(t-\tau_{m}(t)\right)-x\left(t-\tau_{m 2}\right)\right] \\
& -\left[x\left(t-\tau_{m 1}\right)-x\left(t-\tau_{m}(t)\right)\right]^{T} R_{2}\left[x\left(t-\tau_{m 1}\right)-x\left(t-\tau_{m}(t)\right)\right] \\
& -\frac{\tau_{m}(t)-\tau_{m 1}}{\tau_{m 2}-\tau_{m}(t)}\left[x\left(t-\tau_{m}(t)\right)-x\left(t-\tau_{m 2}\right)\right]^{T} R_{2}\left[x\left(t-\tau_{m}(t)\right)-x\left(t-\tau_{m 2}\right)\right] \\
& -\frac{\tau_{m 2}-\tau_{m}(t)}{\tau_{m}(t)-\tau_{m 1}}\left[x\left(t-\tau_{m 1}\right)-x\left(t-\tau_{m}(t)\right)\right]^{T} R_{2}\left[x\left(t-\tau_{m 1}\right)-x\left(t-\tau_{m}(t)\right)\right] \\
\leq & -\left[x\left(t-\tau_{m}(t)\right)-x\left(t-\tau_{m 2}\right)\right]^{T} R_{2}\left[x\left(t-\tau_{m}(t)\right)-x\left(t-\tau_{m 2}\right)\right] \\
& -\left[x\left(t-\tau_{m 1}\right)-x\left(t-\tau_{m}(t)\right)\right]^{T} R_{2}\left[x\left(t-\tau_{m 1}\right)-x\left(t-\tau_{m}(t)\right)\right]
\end{aligned}
$$

Similar to (13), we obtain

$$
\begin{aligned}
-\int_{t-\tau_{p 2}}^{t-\tau_{p 1}}\left(\tau_{p 2}-\tau_{p 1}\right) \dot{y}^{T}(\theta) R_{4} \dot{y}(\theta) d \theta \leq & -\left[y\left(t-\tau_{p}(t)\right)-x\left(t-\tau_{p 2}\right)\right]^{T} R_{4}\left[y\left(t-\tau_{p}(t)\right)-y\left(t-\tau_{p 2}\right)\right] \\
& -\left[y\left(t-\tau_{p 1}\right)-y\left(t-\tau_{p}(t)\right)\right]^{T} R_{4}\left[y\left(t-\tau_{p 1}\right)-y\left(t-\tau_{p}(t)\right)\right] .
\end{aligned}
$$

In addition, for any $\Gamma_{m}=\operatorname{diag}\left(\Gamma_{m 1}, \ldots, \Gamma_{m 2}\right) \geq 0, m=1,2$, the inequality below is established from (5)

$$
-2 \tilde{g}(y(t)) \Gamma_{1}[\tilde{g}(y(t))-N y(t)]-2 \tilde{g}^{T}\left(y\left(t-\tau_{p}(t)\right)\right) \Gamma_{2}\left[\tilde{g}\left(y\left(t-\tau_{p}(t)\right)\right)-N y\left(t-\tau_{p}(t)\right)\right] \geq 0 .
$$

From (9) to (15), we have

$$
\begin{aligned}
\dot{V}(t) \leq & 2 x^{T}(t) P_{1}\left[-K_{m} x(t)+E \tilde{g}\left(y\left(t-\tau_{p}(t)\right)\right)\right]+2 y^{T}(t) P_{2}\left[-K_{p} y(t)+D x\left(t-\tau_{m}(t)\right)\right]+x^{T}(t) G_{1} x(t) \\
& -x^{T}\left(t-\tau_{p}(t)\right) G_{1} x^{T}\left(t-\tau_{m}(t)\right)\left(1-\tau_{m 3}\right)+y^{T}(t) G_{2} y(t)+2 y^{T}(t) M_{1} \tilde{g}(y(t))+\tilde{g}^{T}(y(t)) G_{3} \tilde{g}(y(t)) \\
& -y^{T}\left(t-\tau_{p}(t)\right) G_{2} y\left(t-\tau_{p}(t)\right)\left(1-\tau_{p 3}\right)-2 y^{T}\left(t-\tau_{p}(t)\right) M_{1} \tilde{g}\left(y\left(t-\tau_{p}(t)\right)\right)\left(1-\tau_{p 3}\right) \\
& -\tilde{g}\left(y^{T}\left(t-\tau_{p}(t)\right)\right) G_{3} \tilde{g}\left(y\left(t-\tau_{p}(t)\right)\right)\left(1-\tau_{p 3}\right)+\tau_{m 1}^{2}\left[-K_{m} x(t)+E \tilde{g}\left(y\left(t-\tau_{p}(t)\right)\right)\right]^{T} R_{1}\left[-K_{m} x(t)\right. \\
& \left.+E \tilde{g}\left(y\left(t-\tau_{p}(t)\right)\right)\right]+\left(\tau_{m 2}-\tau_{m 1}\right)^{2}\left[-K_{m} x(t)+E \tilde{g}\left(y\left(t-\tau_{p}(t)\right)\right)\right] R_{2}\left[-K_{m} x(t)+E \tilde{g}\left(y\left(t-\tau_{p}(t)\right)\right)\right] \\
& +\tau_{p 1}^{2}\left[-K_{p} y(t)+D x\left(t-\tau_{m}(t)\right)\right]^{T} R_{3}\left[-K_{p} y(t)+D x\left(t-\tau_{m}(t)\right)\right]+\left(\tau_{p 2}-\tau_{p 1}\right)^{2}\left[-K_{p} y(t)+D x\left(t-\tau_{m}(t)\right)\right]^{T} \\
& \times R_{4}\left[-K_{p} y(t) D x\left(t-\tau_{m}(t)\right)\right]-\left[x(t)-x\left(t-\tau_{m 1}\right)\right]^{T} R_{1}\left[x(t)-x\left(t-\tau_{m 1}\right)\right]-\left[y(t)-y\left(t-\tau_{p 1}\right)\right]^{T} \\
& \times R_{1}\left[y(t)-y\left(t-\tau_{p 1}\right)\right]-\left[x\left(t-\tau_{m}(t)\right)-x\left(t-\tau_{m 2}\right)\right]^{T} R_{2}\left[x\left(t-\tau_{m}(t)\right)-x\left(t-\tau_{m 2}\right)\right] \\
& -\left[x\left(t-\tau_{m 1}\right)-x\left(t-\tau_{m}(t)\right)\right]^{T} R_{2}\left[x\left(t-\tau_{m 1}\right)-x\left(t-\tau_{m}(t)\right)\right]-\left[y\left(t-\tau_{p}(t)\right)-x\left(t-\tau_{p 2}\right)\right]^{T} \\
& \times R_{4}\left[y\left(t-\tau_{p}(t)\right)-y\left(t-\tau_{p 2}\right)\right]-\left[y\left(t-\tau_{p 1}\right)-y\left(t-\tau_{p}(t)\right)\right]^{T} R_{4}\left[y\left(t-\tau_{p 1}\right)-y\left(t-\tau_{p}(t)\right)\right] \\
& -2 \tilde{g}(y(t)) \Gamma_{1}[\tilde{g}(y(t))-N y(t)]-2 \tilde{g}\left(y\left(t-\tau_{p}(t)\right)\right) \Gamma_{2}\left[\tilde{g}\left(y\left(t-\tau_{p}(t)\right)\right)-N y\left(t-\tau_{p}(t)\right)\right] \\
\leq & \zeta^{T} \Phi \zeta+\alpha\left(x^{T}(t) P_{1} x(t)+y^{T}(t) p_{2} y(t)\right) \\
\leq & \zeta^{T} \Phi \zeta+\alpha V(t)
\end{aligned}
$$

where

$$
\begin{gathered}
\zeta^{T}=\left[x^{T}(t), x^{T}\left(t-\tau_{m 1}\right), x^{T}\left(T-\tau_{m}(t)\right), x^{T}\left(t-\tau_{m 2}\right), y^{T}(t), y^{T}\left(t-\tau_{p 1}\right),\right. \\
\left.y^{T}\left(T-\tau_{p}(t)\right), y^{T}\left(t-\tau_{p 2}\right), \tilde{g}(y(t)), \tilde{g}^{T}\left(y\left(t-\tau_{p}(t)\right)\right)\right] .
\end{gathered}
$$

By condition (7), we have

$$
\dot{V}(t) \leq \alpha V(t)
$$


Integrating (17) from $t_{k}$ to $t$, we get

$$
V(t) \leq e^{\alpha}\left(t-t_{k}\right) V(t)
$$

Since the Assumption2, we have

$$
\begin{aligned}
V(t) & \leq e^{\alpha\left(t-t_{k}\right)} V\left(t_{k}^{-}\right) \\
& \leq e^{\alpha\left(t-t_{k-1}\right)} V\left(t_{k-1}\right) \\
& \leq e^{\alpha\left(t-t_{0}\right)} V\left(t_{0}\right) \\
& \leq e^{\alpha T} V(\varphi(t), \psi(t))
\end{aligned}
$$

In another area, by the Assumption1, we get

$$
\begin{aligned}
V(\varphi(t), \psi(t))= & \varphi^{T}(t) P_{1} \varphi(t)+\psi^{T}(t) P_{2} \psi(t) \\
& +\int_{t-\tau_{m}(t)}^{t} \varphi^{T}(s) G_{1} \varphi(s) d s+\int_{t-\tau_{p}(t)}^{t}\left[\psi^{T}(s), g^{T}(\psi(s))\right]\left[\begin{array}{cc}
G_{2} & M_{1} \\
* & G_{3}
\end{array}\right] \times\left[\begin{array}{c}
\psi(s) \\
g(\psi(s))
\end{array}\right] d s \\
& +\int_{-\tau_{m 1}}^{0} \int_{t+\theta}^{t} \tau_{m 1} \dot{\varphi}^{T}(s) R_{1} \dot{\varphi}(s) d s d \theta+\int_{-\tau_{m 2}}^{-\tau_{m 1}} \int_{t+\theta}^{t}\left(\tau_{m 2}-\tau_{m 1}\right) \dot{\varphi}^{T}(s) R_{2} \dot{\varphi}(s) d s d \theta \\
& +\int_{-\tau_{p 1}}^{0} \int_{t+\theta}^{t} \tau_{p 1} \dot{\psi}^{T}(s) R_{3} \dot{\psi}(s) d s d \theta+\int_{-\tau_{p 2}}^{-\tau_{p 1}} \int_{t+\theta}^{t}\left(\tau_{p 2}-\tau_{p 1}\right) \dot{\psi}^{T}(s) R_{4} \dot{\psi}(s) d s d \theta, \\
\leq & \lambda_{\max }\left(Q^{-1} P_{1}\right)\|\varphi(t)\|^{2}+\lambda_{\max }\left(Q^{-} 1 P_{2}\right)\|\psi(t)\|^{2}+\tau_{m 2} \lambda_{\max }\left(Q^{-1} G_{1}\right)\|\varphi(t)\|^{2} \\
& +\tau_{p 2}\left(\lambda_{\max }\left(Q^{-1} G_{2}\right)\|\psi(t)\|^{2}+\eta^{2} \lambda_{\max }\left(Q^{-1} G_{3}\right)\|\psi(t)\|^{2}+2 \eta \lambda_{\max }\left(Q^{-1} M_{1}\right)\right)\|\psi(t)\|^{2} \\
& +1 / 2\left[\tau_{m 1}^{3} \lambda_{\max }\left(Q^{-1} R_{1}\right)+\left(\tau_{m 2}-\tau_{m 1}\right)^{3} \lambda_{\max }\left(Q^{-1} R_{2}\right)\right]\|\varphi(t)\|^{2} \\
& +1 / 2\left[\tau_{p 1}^{3} \lambda_{\max }\left(Q^{-1} R_{3}\right)+\left(\tau_{p 2}-\tau p 1\right)^{3} \lambda_{\max }\left(Q^{-1} R_{4}\right)\right]\|\varphi(t)\| \\
\leq & {\left[\lambda_{\max }\left(Q^{-1} P_{1}\right)+\tau_{m 2} \lambda_{\max }\left(Q^{-1} G_{1}\right)+1 / 2\left[\tau_{m 1}^{3} \lambda_{\max }\left(Q^{-1} R_{1}\right)+\left(\tau_{m 2}-\tau_{m 1}\right)^{3} \lambda_{\max }\left(Q^{-1} R_{2}\right)\right]\right]\|\varphi(t)\|^{2} } \\
& +\left[\tau_{p 2}\left(\lambda_{\max }\left(Q^{-1} G_{2}\right)+\eta^{2} \lambda_{\max }\left(Q^{-1} G_{3}\right)+2 \eta \lambda_{\max }\left(Q^{-1} M_{1}\right)\right)+\lambda_{\max }\left(Q^{-1} P_{2}\right)\right. \\
& \left.+1 / 2\left[\tau_{p 1}^{3} \lambda_{\max }\left(Q^{-1} R_{3}\right)+\left(\tau_{p 2}-\tau p 1\right)^{3} \lambda_{\max }\left(Q^{-1} R_{4}\right)\right]\right]\|\psi(t)\|^{2} \\
\leq & \lambda_{1}\left(\|\varphi(t)\|^{2}+\|\psi(t)\|^{2}\right)
\end{aligned}
$$

In the meanwhile,

$$
V(t) \geq \min \left[\lambda_{\min }\left(P_{1}\right)\|x(t)\|^{2}+\lambda_{\min }\left(P_{2}\right)\|y(t)\|^{2}\right] \geq \lambda_{2}\left(\|x(t)\|^{2}+\|y(t)\|^{2}\right)
$$

From (17) and (18) we obtain

$$
\begin{aligned}
\|x(t)\|^{2}+\|y(t)\|^{2} & \leq \frac{1}{\lambda_{2}} V(t) \leq \frac{1}{\lambda_{2}} e^{\alpha T} V(\varphi(t), \psi(t)) \\
& \leq \frac{1}{\lambda_{2}} e^{\alpha T} \lambda_{1} c_{1} \leq \frac{\lambda_{1}}{\lambda_{2}} e^{\alpha T} c_{1} \leq c_{2}
\end{aligned}
$$

Thus we complete the proof.

\section{Numerical Examples}

In this section, we put forward an example to explain the significance of our results. Let us consider a GRNs model of this system.

$$
\left\{\begin{array}{c}
\dot{x}(t)=-K_{m} x(t)+E \tilde{g}\left(y\left(t-\tau_{p}(t)\right)\right) \\
\dot{y}(t)=-K_{p} x(t)+D x\left(t-\tau_{m}(t)\right) \\
\triangle x(t)=J_{k}\left(x\left(t_{k}^{-}\right)\right) \\
\triangle y(t)=H_{k}\left(y\left(t_{k}^{-}\right)\right)
\end{array}\right.
$$


where $K_{m}=\operatorname{diag}[4,4,4], K_{p}=\operatorname{diag}[4,4,4], J_{k}=\operatorname{diag}[-0.75,-0.75,-0.75], H_{k}=\operatorname{diag}[-0.95,-0.95,-0.95]$ $D=\operatorname{diag}[0.5,0.4,0.6]$

$$
E=\left(\begin{array}{lll}
0 & 1 & 0 \\
0 & 0 & 1 \\
1 & 0 & 0
\end{array}\right)
$$

The gene regulation function is taken as $g(x)=x^{2} /\left(1+x^{2}\right), N=\operatorname{diag}(0.65,0.65,0.65), t_{k}=2.5 k, T=5, c_{1}=$ $2, c_{2}=1.5, \alpha=0.02$, the time delay $\tau_{p}(t), \tau_{m}(t)$ are assumed to be:

$$
\tau_{p}(t)=0.6+0.4 \sin (t), \tau_{m}(t)=0.3+0.1 \cos (t)
$$

we obtain the parameters below:

$$
\tau_{p 1}=0.2, \tau_{p 2}=1, \tau_{p 3}=0.4, \tau_{m 1}=0.2, \tau_{m 2}=0.4, \tau_{m 3}=0.1
$$

We calculate that the following feasible solutions for LMI (7) :

$$
\begin{aligned}
& P_{1}=\left(\begin{array}{lll}
1.7556 & 0.0199 & 0.0199 \\
0.0199 & 1.7529 & 0.0199 \\
0.0199 & 0.0199 & 1.7585
\end{array}\right), \quad P_{2}=\left(\begin{array}{ccc}
1.0342 & -0.0006 & -0.0007 \\
-0.0006 & 0.9145 & -0.0006 \\
-0.0007 & -0.0006 & 1.1411
\end{array}\right) \\
& G_{1}=\left(\begin{array}{ccc}
5.7466 & 0.0419 & 0.0418 \\
0.0419 & 5.7240 & 0.0418 \\
0.0418 & 0.0418 & 5.7720
\end{array}\right), \quad G_{2}=\left(\begin{array}{ccc}
8.2762 & -0.0003 & -0.0003 \\
-0.0003 & 8.1893 & -0.0003 \\
-0.0003 & -0.0003 & 8.3543
\end{array}\right) \\
& G_{3}=\left(\begin{array}{lll}
5.2570 & 0.0003 & 0.0003 \\
0.0003 & 5.2570 & 0.0003 \\
0.0003 & 0.0003 & 5.2570
\end{array}\right), \quad R_{1}=\left(\begin{array}{lll}
3.0654 & 0.0739 & 0.0736 \\
0.0739 & 3.0675 & 0.0737 \\
0.0736 & 0.0737 & 3.0631
\end{array}\right) \\
& R_{2}=\left(\begin{array}{lll}
2.0856 & 0.0119 & 0.0119 \\
0.0119 & 2.0778 & 0.0119 \\
0.0119 & 0.0119 & 2.0943
\end{array}\right), \quad R_{3}=\left(\begin{array}{lll}
3.4685 & 0.0000 & 0.0000 \\
0.0000 & 3.4540 & 0.0000 \\
0.0000 & 0.0000 & 3.4817
\end{array}\right) \\
& R_{4}=\left(\begin{array}{ccc}
1.0090 & 0.0004 & 0.0006 \\
0.0004 & 1.0915 & 0.0005 \\
0.0006 & 0.0005 & 0.9355
\end{array}\right), \quad M_{1}=\left(\begin{array}{ccc}
-1.6161 & 0.0000 & 0.0000 \\
0.0000 & -1.6161 & 0.0000 \\
0.0000 & 0.0000 & -1.6161
\end{array}\right) \\
& \Lambda_{1}=\left(\begin{array}{lll}
5.8675 & 0.0000 & 0.0000 \\
0.0000 & 5.8675 & 0.0000 \\
0.0000 & 0.0000 & 5.8675
\end{array}\right), \quad \Lambda_{2}=\left(\begin{array}{lll}
3.1570 & 0.0000 & 0.0000 \\
0.0000 & 3.1569 & 0.0000 \\
0.0000 & 0.0000 & 3.1568
\end{array}\right)
\end{aligned}
$$

The initial condition is $x(0)=[0.2,0.2,0.3]^{T}, y(0)=[0.3,0.2,0.5]^{T}$. The simulation results of the mRNA concentrations are shown in Figures 1. The simulation results of the protein concentrations are shown in Figures 2. So, the system of GRNs with impulsive effects is finite-time stable.

\section{Conclusions}

We considered the problem of finite-time stability for GRNs with impulsive effects in this paper. The sufficient conditions were provided for the system to be finite-time stable based on lyapunov function method. We represent a numerical example to shown the proposed theoretical results. In the future, we will further study the finite-time stability for the genetic regulatory with noise perturbations([21, 22, 23]), and the dynamics of stochastic GRNS with mixed time-delays([24, 25]). 


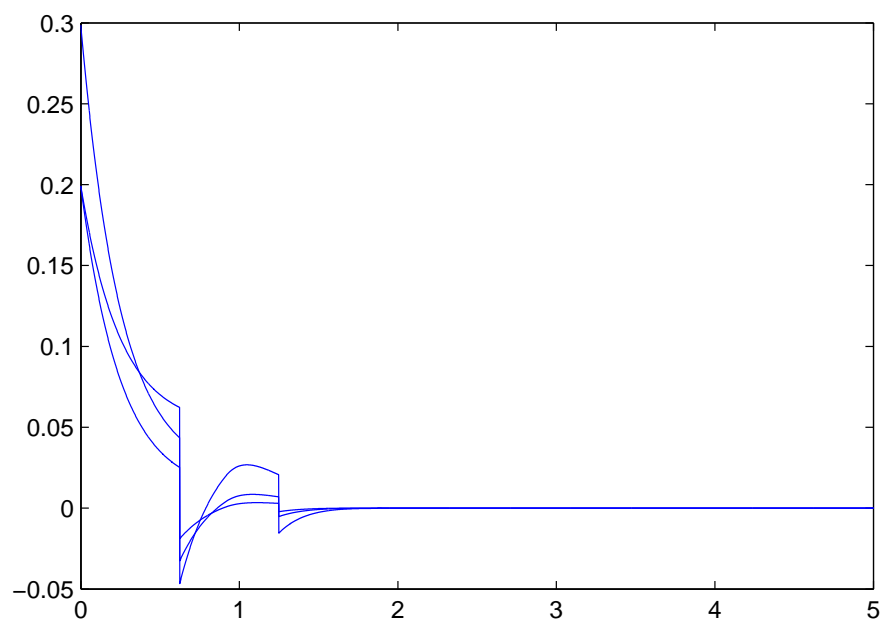

Figure 1: mRNA concentrations $x(t)$.

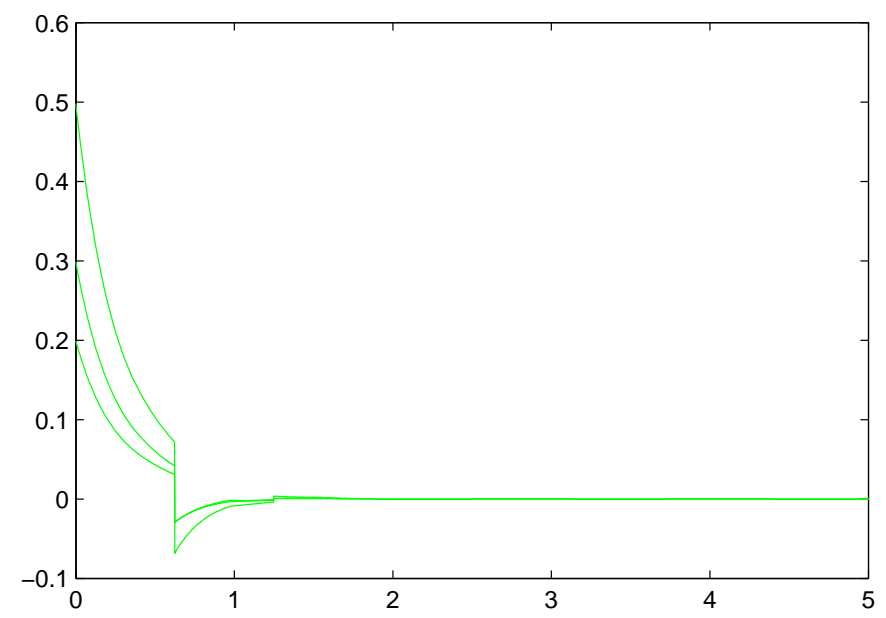

Figure 2: Protein concentrations $y(t)$. 


\section{Acknowledgement}

This work was supported in part by the National Science Foundation of China ( Nos. 61273012, 61304023 and 61403179), by the Project of Shandong Province Higher Educational Science and Technology Program (No. J12LI58), and by Applied Mathematics Enhancement Program of Linyi University.

[1] M. Takada, Y. Hori. Comments and corrections on "Stability of genetic regulatory networks with time delay", IEEE Transactions on Circuits and Systems-I:Regular Papers, 61(2014): 2771-2774.

[2] F. Wu. Delay-independent stability of genetic regulatory networks, IEEE Transactions on Circuits and Systems, 22(2011): $1685-1693$.

[3] J. Cao, F. Ren. Exponential stability of discrete-time genetic regulatory networks with delays, IEEE Transactions on Circuits and Systems, 19(2008): 520-523.

[4] J. Hu, J. Liang, J. Cao. Stability analysis for genetic regulatory networks with delays: The continuous-time case and the discrete-time case, Applied Mathematics and Computation, 220(2013): 507-517

[5] J. Liang, J. Lam. Robust state estimation for stochastic genetic regulatory networks, International Journal of Systems Science, 41(2010): 47-63.

[6] L. Tian, Z. Shi, L. Liu, F. Wu. M-matrix-based stability conditions for genetic regulatory networks with time-varying delays and noise perturbations, Institution of Engineering and Technology, 7(2013): 214-222.

[7] T. S. Gardner, C. R. Cantor, J. J. Collins. Construction of genetic toggle switch in Escherichia Coli, Nature, 403(2000): 339-342.

[8] M. B. Elowitz, S. Leiber. A synthetic oscillatory network of transcription regulators, Nature, 403(2000): $335-338$.

[9] L. Chen, K. Aihara. Stability of genetic regulatory networks with time delay, IEEE Transactions on Circuits and systems-I:Fundamental Theory and Applications, 49(2002): 602-608.

[10] Y. Wang, Z. Wang, J. Liang. On Robust Stability of Stochastic Genetic Regulatory Networks With Time Delays: A Delay Fractioning Approach, IEEE Transactions on Systems, Man, and Cybernetics, Part B-Cybernetics, 40(2010): 729-740.

[11] J. Liang, J. Lam, Z. Wang. State estimation for Markov-type genetic regulatory networks with delays and uncertain mode transition rates, Physics Letters A, 373(2009): 4328-4337.

[12] B. Lv, J. Liang, J. Cao. Robust distributed state estimation for genetic regulatory networks with markovian jumping parameters, Communications in Nonlinear Science and Numerical Simulation, 16(2011): 4060-4078.

[13] B. Shen, Z. Wang, J. Liang. Sampled-data H-infinity filtering for stochastic genetic regulatory networks, International Journal of Robust and Nonlinear Control, 21(2011): 1759-1777.

[14] Y. Yang, J. Liang, J. Cao. Stability analysis for switched genetic regulatory networks: An average dwell time approach, Journal of the Franklin Institute-Engineering and Applied Mathematics. 348(2011): 2718-2733.

[15] S. Zhao, J. Sun, L. Liu. Finite-time stability of linear time-varying singular systems with impusive effects. International Journal of Control, 81(2008): 1824-1829

[16] J. Xu, J. Sun. Finite-time filtering for discrete-time linear impulsive sysytems. Signal Processing, 92(2012): 2718-2722.

[17] J. Xu, J. Sun. Finite-time stability of nonlinear switched impulsive systems. International Journal of Systems Science, 44(2013): 889-895.

[18] A. Liu, Y. Li, D. Zhang, W. Zhang. Finite-time $H_{\infty}$ control for discrete-time genetic regulatory networks with random delays and partly unknown transition probabilities, Journal of the Franklin Institute, 350(2013): 1944-1961.

[19] L. Yin. Finite-time stability analysis of switched Genetic Regulatory Networks, Journal of Applied Mathematics, 2014(2014): 1-11.

[20] J. Zhou, S. Xu, H. Shen. Finite-time robust stochastic stability of uncertain stochastic delayed reaction-diffusion genetic regulatory networks, Neurocomputing, 74(2011): 2790-2796.

[21] Z. Wang, X. Liu, Y. Liu, J. Liang, V. Vinciotti. Ant Extended Kalman Filtering Approach to Modeling Nonlinear Dynamic Gene Regulatory Networks via Short Gene Expression Time Series, IEEE-ACM Transactions on Computational Biology and Bioinformatics, 6(2009): 410-419.

[22] Z. Wang, H. Wu, J. Liang. On Modeling and State Estimation for Genetic Regulatory Networks With Polytopic Uncertainties, IEEE Transactions on Nanobioscience, 12(2013): 13-20.

[23] W. Wang, X. Liu, Y. Li. Set-membership filtering for genetic regulatory networks with missing values, Neurocomputing, 175(2016): 466-472.

[24] X. Zhang, Y. Han, L. Wu. M-matrix-based globally asymptotic stability criteria for genetic regulatory networks with time-varying discrete and unbounded distributed delays, Neurocomputing, 174(2016): 1060-1069

[25] J. Hu, J. Liang, J. Cao. Stabilization of genetic regulatory networks with mixed time-delays: an adaptive control approach. IMA Journal of Mathematical Control and Information, 32(2015): 343-358 